\title{
Separation of Enantiomers through Local Vorticity: A Screw Model Mechanism
}

\author{
Anderson D. S. Duraes and J. Daniel Gezelter* \\ 251 Nieuwland Science Hall, Department of Chemistry and Biochemistry, \\ University of Notre Dame, Notre Dame, Indiana 46556, United States \\ E-mail: gezelter@nd.edu
}




\begin{abstract}
We present a model to explain the mechanism behind enantiomeric separation under either shear flow or local rotational motion in a fluid. Local vorticity of the fluid imparts molecular rotation that couples to translational motion, sending enantiomers in opposite directions. Translation-rotation coupling of enantiomers is explored using the molecular hydrodynamic resistance tensor, and a molecular equivalent of the pitch of a screw is introduced to describe the degree of translation-rotation coupling. Molecular pitch is a structural feature of the molecules and can be easily computed, allowing rapid estimation of the pitch of 85 drug-like molecules. Simulations of model enantiomers in a range of fluids such as $\Lambda$ - and $\Delta-\left[\mathrm{Ru}(\mathrm{bpy})_{3}\right] \mathrm{Cl}_{2}$ in water and $(R, R)$ - and $(S, S)$-atorvastatin in methanol support predictions made using molecular pitch values. A competition model and continuum drift diffusion equations are developed to predict separation of realistic racemic mixtures. We find that enantiomeric separation on a centimeter length scale can be achieved in hours, using experimentally-achievable vorticities. Additionally, we find that certain achiral objects can also exhibit a non-zero molecular pitch.
\end{abstract}




\section{Introduction}

Enantiomers of chiral molecules are non-superposable mirror images with the same structural formula. ${ }^{1,2}$ In achiral environments, enantiomers have identical physical and chemical properties, and this prevents separation by classical methods. ${ }^{2-4}$ For example, crystallization and filtration (separation by solubility), ${ }^{5}$ distillation (separation by boiling point) ${ }^{5}$ and standard achiral chromatography (separation by interaction with a stationary phase and a solvent) ${ }^{5}$ are unable to resolve enantiomers.

The current methods to resolve enantiomers are expensive and not universal. ${ }^{2-4,6}$ These methods typically require changes in synthetic pathways or separation in chiral environments. Synthetic methods will often utilize acid-base reactions that form diastereomers, while subsequent reactions will recover the pure enantiomers from the resolved diastereomers. This allows resolution of the enantiomers via classical techniques due to distinct physical and chemical properties of intermediates. $^{2-4}$

There has also been significant progress on separating enantiomers once they have been formed by their primary synthetic reactions. In this case, the enantiomers can be resolved through chiral environments. Chiral environments often involve chiral chromatography ${ }^{2,4,7}$ where enantiomers have distinct intermolecular interactions with a chiral stationary phase or with a chiral solvent. ${ }^{2,4,7}$

In this paper, we develop an explanatory "screw" model for predicting the shear-flow separation of enantiomers using a set of hydrodynamic calculations on the structures of the molecules. Although the fluid itself may be achiral, shear vorticity of the fluid introduces a dynamic chiral environment that can induce separation. Shear vorticity is the rotation of an object induced by a velocity gradient in a fluid undergoing shear flow. ${ }^{8,9}$ The potential separation of enantiomers by achiral fluid flow was reported by Howard et al. ${ }^{10,11}$ Inside a rotating drum, Howard and coworkers suspended dextro-tartaric acid crystals in Isopar H (isoparaffinic hydrocarbons), and observed pure macroscopic enantiomers moving in specific directions depending on the Isopar $\mathrm{H}$ flow. ${ }^{10}$

Howard et al.'s idea ${ }^{10,11}$ has been extended to separating biological and manufactured chiral 
objects (not molecules) at the mili, ${ }^{12,13}$ micro ${ }^{14-17}$ and nano ${ }^{18}$ scale. These observations contradict a simple theoretical model of Tencer and Bielski, ${ }^{19}$ which suggests an impractical amount of time required to resolve micro-scale or smaller chiral objects. With these experimental and theoretical discrepancies, Hermans et al. ${ }^{20}$ have recently emphasized the necessity of theoretical studies at the small scales, particularly on real molecules with all intermolecular interactions treated in a realistic manner.

\section{Formalism}

We first describe how hydrodynamics enters molecular simulations. When a body is moving through an implicit, dense fluid, we often adopt Langevin Dynamics (LD) to describe the motion of the body,

$$
m \dot{\mathbf{v}}(t)=-\nabla U-\xi \mathbf{v}(t)+\mathbf{R}(t),
$$

where $m$ and $\mathbf{v}$ are the mass and velocity of the body, $\nabla U$ is the gradient of the potential energy, which provides the 'system' force on the body, and frictional forces $(-\xi \mathbf{v})$ and random forces $(\mathbf{R})$ are present due to the interactions with the implicit fluid.

In a typical LD simulation, the frictional forces are modeled using Stokes' law, $\xi \approx 6 \pi \eta \sigma$, where $\eta$ is the viscosity of the implicit fluid, and $\sigma$ is the hydrodynamic radius of the body. ${ }^{21,22}$ Additionally, the friction and random forces are tied together by the second fluctuation-dissipation theorem,

$$
\left\langle\mathbf{R}(t) \cdot \mathbf{R}\left(t^{\prime}\right)\right\rangle=2 k_{B} T \xi \delta\left(t-t^{\prime}\right),
$$

where the random forces at different times are uncorrelated in the high friction limit.

To describe the motion of a complex rigid body, which has both linear and angular velocities, we must broaden the Langevin approach to include both frictional forces and torques. These can 
be included through a resistance tensor, ${ }^{23,24}$

$$
\left(\begin{array}{c}
\mathbf{F}_{f} \\
\tau_{f}
\end{array}\right)=-\left(\begin{array}{cc}
\Xi^{\mathrm{tt}} & \Xi^{\mathrm{rt}} \\
\Xi^{\mathrm{tr}} & \Xi^{\mathrm{rr}}
\end{array}\right)\left(\begin{array}{l}
\mathbf{v} \\
\omega_{b}
\end{array}\right),
$$

that mediates the relationship among frictional force $\left(\mathbf{F}_{f}\right)$, torque $\left(\tau_{f}\right)$, velocity $(\mathbf{v})$ and bodyfixed angular velocity $\left(\omega_{b}\right)$. The resistance tensor is composed of $3 \times 3$ blocks, where $\Xi^{\text {tt }}$ is the translational and $\Xi^{\text {rr }}$ is the rotational resistance tensor, while $\Xi^{\text {tr }}$ is the translation-rotation and $\Xi^{\mathrm{rt}}=\left(\Xi^{\mathrm{tr}}\right)^{T}$ is the rotation-translation coupling tensor.

If a fluid is moving past a body with velocity, $\mathbf{v}$, the same resistance tensor in Eq. (1) also governs the forces (and torques) experienced by the body, although the sign of the velocity is reversed. Similarly, if the surrounding fluid is exhibiting a vortex flow, this same tensor will govern torques (and forces) on the body. To illustrate this point, consider the 'propeller'-shaped molecules, $\Lambda$-tris(2,2'-bipyridine) ruthenium (II) and $\Delta$-tris(2,2'-bipyridine) ruthenium (II) shown in Fig. 1. If these molecules are placed in a moving fluid, they begin rotating in opposite directions in their body-fixed frames. However, if the surrounding fluid is experiencing a vortex flow rotating around the molecules, they will begin translating in opposite directions. It is exactly this property which can be exploited to separate enantiomers.

Rotational motion in a fluid is not limited to portions of the fluid that are swirling around a vortex line. It can also be observed in regions where a fluid is undergoing simple linear shear flow. To measure local rotation in a fluid, we use vorticity, ${ }^{21,22,25,26}$

$$
\omega=\nabla \times \mathbf{v}(\mathbf{r})
$$

where $\mathbf{v}(\mathbf{r})$ is the local velocity field in the fluid, and $\omega \neq 0$ indicates rotation.

For a rigid body, a general relationship between vorticity $(\omega)$ and angular velocity $\left(\omega_{b}\right)$ is known. ${ }^{22,25,26}$ A fluid flow with vorticity $\omega \neq 0$ induces the rotation of the rigid body, which then acquires an angular velocity $\omega_{b}=\omega / 2$. 


\section{The Screw Model}

A screw is a simple chiral object that couples rotation around one axis with displacement along that axis. This displacement is perpendicular to the plane of rotation and is characterized by the screw's pitch (or lead). Here we define pitch $(P)$ as the perpendicular distance advanced by a screw in a $2 \pi$-revolution (See Fig. 1). In a lab-fixed frame, left- and right-handed screws have pitches with the same magnitude, but with flipped signs, rotating in opposite directions to do the same task. $23,27-29$

This concept can be easily extended to chiral molecules by considering the translation-rotation coupling tensor in Eq. (1). In a medium which induces rotation of molecules (as in a vortex flow), chiral molecules will translate in opposite directions. As a consequence, this asymmetry may enable the resolution of the enantiomers. ${ }^{23,28}$

In the following sections, we develop a hydrodynamic framework to rapidly estimate molecular pitches, as well a competition model for the separation of chiral molecules in solution. We note that the molecules do not need to display screw-like geometries for this separation method to be effective.

Calculation of the resistance tensor, $\Xi$, for arbitrary molecular geometries was pioneered by Brenner, ${ }^{23}$ and significantly improved for Brownian dynamics simulations by García de la Torre, whose group introduced a "Rough Shell” approach using small, non-overlapping beads (or spheres) to represent the molecular structure. ${ }^{24,30,31}$ Recent work has extended this model for Langevin dynamics, ${ }^{32}$ and has allowed the use of overlapping beads. ${ }^{33}$ In the Supporting Information, we develop more fully the calculation of the molecular resistance tensors, but we note here that this is a straightforward calculation that requires only the positions and sizes of the atoms in a molecule, and the viscosity of the surrounding fluid.

In Eq. (1), chiral molecules have contributions in the translation-rotation $\left(\Xi^{\mathrm{tr}}\right)$ and rotationtranslation $\left(\Xi^{\mathrm{rt}}\right)$ coupling tensor. Normally, these tensors depend on the origin of the coordinate system, but at the center of resistance, they both become symmetric. For chiral molecules, the eigenvalues of $\Xi^{\text {tr }}$ have the same magnitudes, but flip signs for the two mirror images. Using 

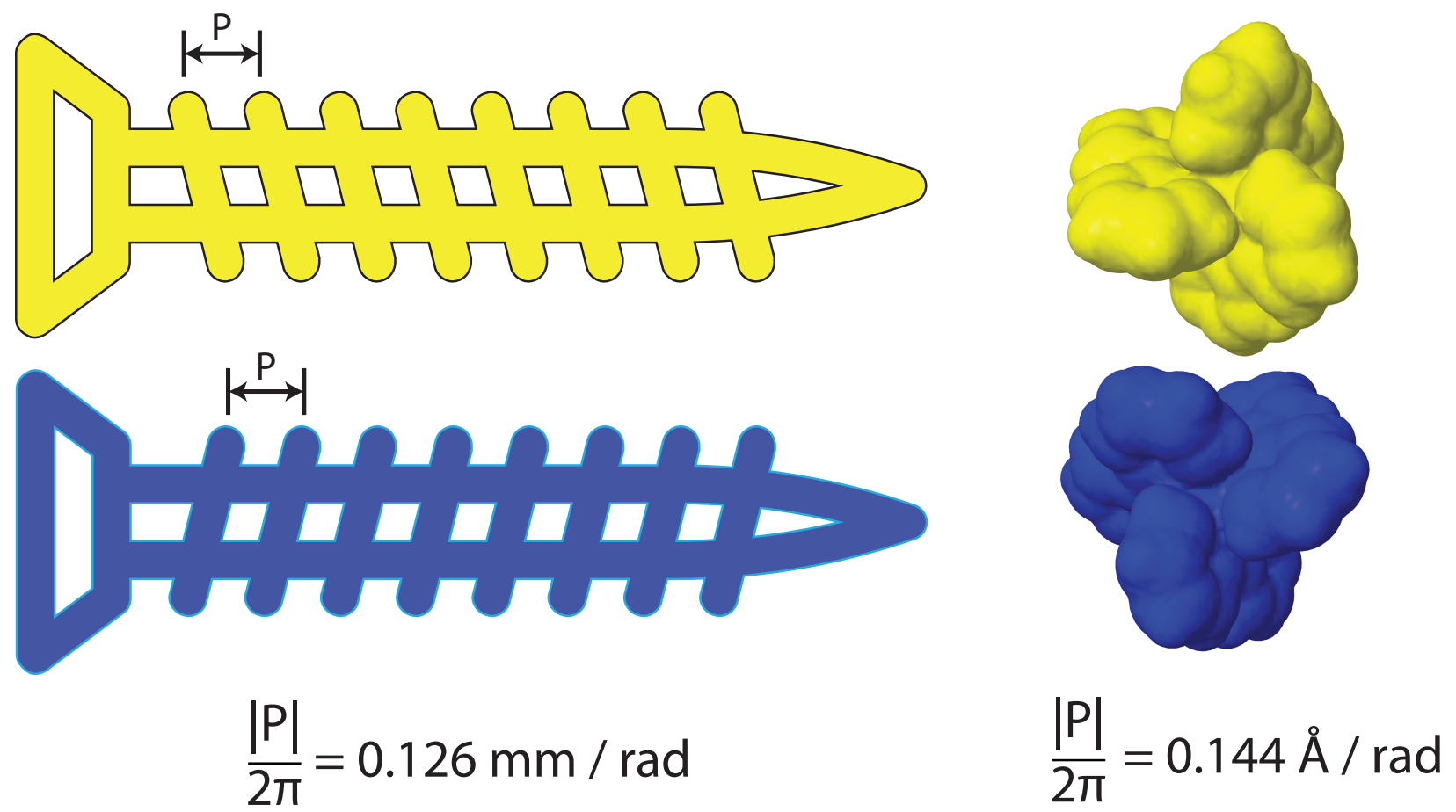

Figure 1: The screw model assigns a pitch $(P)$ to enantiomeric molecules based on rotational invariants of the resistance tensor in Eq. (1). This is a direct analogy to the pitch of regular screws. A typical wood screw might have a pitch measured in mm per radian of rotation, while the $\left[\mathrm{Ru}(\mathrm{bpy})_{3}\right]^{2+}$ ions have a pitch of $0.144 \AA$ per radian of rotation. Screws are chiral objects, and a full $(2 \pi)$ rotation of the enantiomeric screws (or molecules) will separate them by $2 \times|P|$. 
rotational invariants for both $\Xi^{\text {tr }}$ and $\Xi^{\text {tt }}$, we have developed the molecular equivalent of the pitch for a screw or propeller travelling through a medium,

$$
\frac{|P|}{2 \pi}=\sqrt{\frac{\sum_{i}\left(\lambda_{i}^{\mathrm{tr}}\right)^{2}}{\sum_{i}\left(\lambda_{i}^{\mathrm{tt}}\right)^{2}}}
$$

where $\left(\lambda_{i}^{\mathrm{tr}}, \lambda_{i}^{\mathrm{tt}}\right)$ are the $i^{\mathrm{th}}$ eigenvalues of the translation-rotation and translational resistance tensors, respectively. Because $\lambda_{i}^{\text {tr }}$ and $\lambda_{i}^{\text {tt }}$ are linearly proportional to viscosity, the pitch in Eq. (3) has no viscosity dependence, as it is a structural feature of the molecule.

We have calculated the pitch for a large range of common drug molecules ${ }^{34}$ which contain chiral centers. Pitches for selected molecules are shown in table 1. Pitch data for 78 additional chiral drug molecules is provided in the Supporting Information.

Table 1: Molecular pitches for some common enantiomeric molecules calculated using two hydrodynamic models. The rough shell model used non-overlapping beads of radius 0.1 $\AA$, while the overlapping bead model used atom-centered beads with van der Waals radii appropriate for each atom. Molecular pitch values are reported in A / rad. Pitch data on 78 additional molecules are provided in the Supporting Information.

\begin{tabular}{ccc|cc}
\hline \multicolumn{3}{c|}{ Molecule } & \multicolumn{2}{c}{ Molecular Pitch, $|P| / 2 \pi$} \\
\hline Common Name & Generic Name & CAS Number & Rough Shell & Overlapping Bead Model \\
\hline Aricept & Donepezil & $120014-06-4$ & 0.212 & 0.267 \\
Casodex & Bicalutamide & $90357-06-5$ & 0.212 & 0.246 \\
Lipitor & Atorvastatin & $124523-00-5$ & 0.095 & 0.125 \\
Nexium & Esomeprazole & $119141-88-7$ & 0.124 & 0.145 \\
Paxil & Paroxetine & $61869-08-7$ & 0.125 & 0.159 \\
Singulair & Montelukast & $158966-92-8$ & 0.301 & 0.325 \\
Zoloft & Sertraline & $79617-96-2$ & 0.101 & 0.110 \\
& & & 0.144 \\
$(\Lambda$ or $\Delta)-[\text { Ru(bpy) }]_{3}^{2+}$ & & 0.128 & 0.222 \\
$(S)-(-)-1,1^{2}-$ binaphthyl-2,2'-diamine & $18531-95-8$ & 0.175 & 0.0129 \\
$\mathrm{CHBrClF}$ & $593-98-6$ & 0.0092 & \\
\hline \hline
\end{tabular}




\section{Molecular Simulations of Shear-Flow Separation of Enantiomers}

To test the screw model, we have simulated the shear-flow separation of a set of chiral molecules, including the tris(2,2'-bipyridine) ruthenium (II) enantiomers: ${ }^{35,36} \Lambda$ - $\left[\mathrm{Ru}(\mathrm{bpy})_{3}\right]^{2+}$ and $\Delta$-[Ru(bpy)$\left.)_{3}\right]^{2+}$ in both Lennard-Jones argon and SPC/E water ${ }^{37}$ solvents, with $\mathrm{Cl}^{-}$as the counterion. We also explored enantiomeric separation of the $(R)$ and $(S)$ enantiomers of 1,1'-binaphthyl-2,2'-diamine in argon and benzene solvents, and separation of atorvastatin in methanol. As a final test of enantiomeric separation, we studied a neat, racemic mixture of bromochlorofluoromethane, $\mathrm{CHBrClF}$. For argon solvents, simulations were done at $119.8 \mathrm{~K}$, while for all other solvents, the simulations were done at $298 \mathrm{~K}$ and $1 \mathrm{~atm}$. The solvents were chosen because they are either simple monatomic (LJ Argon), or common polar (SPC/E water and methanol), or non-polar (benzene) solvents.

All simulations were done in regions of linear, laminar shear flow, which was generated using the velocity shearing and scaling variant of reverse nonequilibrium molecular dynamics (VSSRNEMD) $)^{38}$ in a simulation cell with periodic boundary conditions. Details of the simulation parameters are provided in the Supporting Information.

RNEMD Momentum Flux $j_{z}\left(p_{x}\right)=$ shear stress $\left(\tau_{z x}\right)$

System response creates two vortex flows
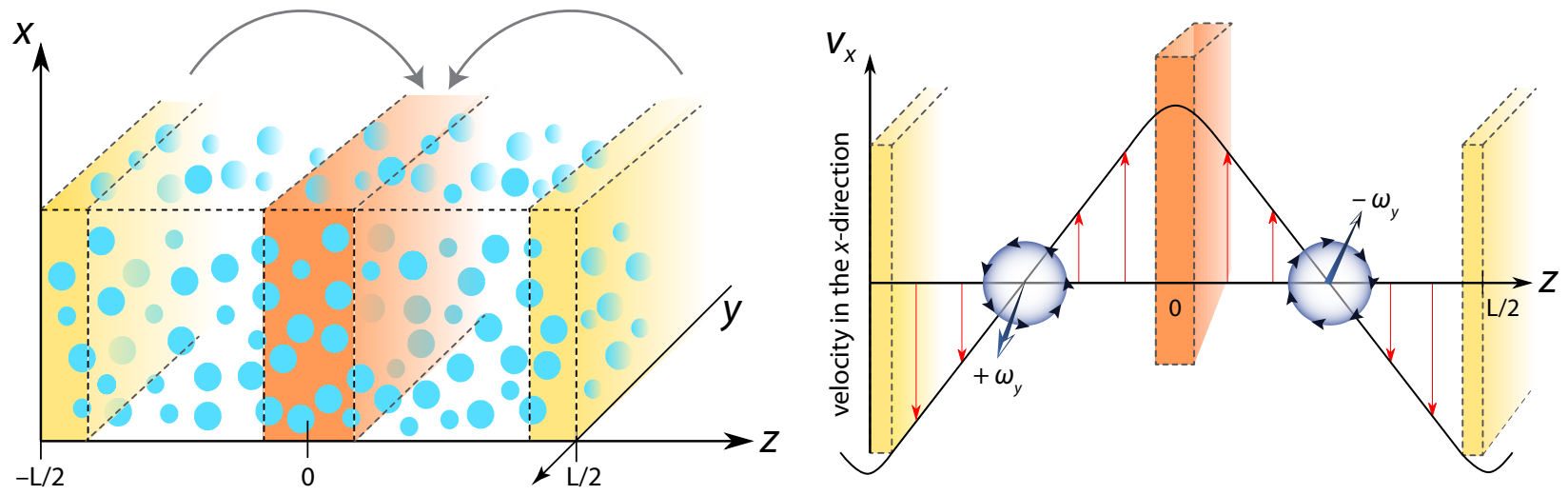

Figure 2: Left: Velocity shearing and scaling reverse non-equilibrium molecular dynamics (VSSRNEMD) imposes a momentum flux, $j_{z}\left(p_{x}\right)$ between two regions (orange and yellow). This is equivalent to a shear stress, $\tau_{z x}$. Right: the fluid responds by creating a velocity profile along the $z$ axis. At low flux, the regions between the orange and yellow slabs (colorless regions) experience a constant velocity gradient, and therefore a constant vorticity, $+\omega_{y}$ or $-\omega_{y}$, which points in opposite directions on either side of the simulation cell. 
Consider the orthorhombic, periodic simulation box in Fig. 2, where the blue circles represent molecules (either solvent or solute). VSS-RNEMD ${ }^{38}$ is a simulation method which imposes an unphysical momentum flux, $j_{z}\left(p_{x}\right)$, between the box's orange and yellow regions. This momentum flux is functionally equivalent to a shear stress, $\tau_{z x}$ on the system. For most fluids, Newton's law connects the shear stress to the shear viscosity, ${ }^{21,22,25,39}$

$$
\tau_{z x}=\eta\left(\frac{\partial v_{x}}{\partial z}\right)
$$

where $v_{x}$ is the $x$-component of the fluid's velocity that evolves in response to the shear stress.

Because simulation cells do not have real, solid slabs to pull, VSS-RNEMD ${ }^{38}$ employs a set of velocity shearing and scaling moves on the atoms inside the orange and yellow regions (Fig. 2). These moves are applied on a periodic basis and the size of the scaling or shearing moves is set by the imposed momentum $\left(j_{z}\left(p_{x}\right)\right)$ or kinetic energy flux. There are additional constraints on the shearing and scaling variables that conserve kinetic energy as well as total linear momentum, so the VSS-RNEMD moves explore (non-equilibrium) portions of the microcanonical (NVE) ensemble. In practice, VSS-RNEMD can also be applied simultaneously with most constant temperature (NVT) or pressure (NPT) integrators.

In a typical RNEMD simulation, the system responds to the momentum exchange between the yellow and orange regions (Fig. 2) with a linear response in the colorless regions, where the system response is recorded. For RNEMD simulations with momentum exchange, the system develops two opposing velocity gradients between the exchange regions. In Fig. 2, the left region has a positive and nearly constant $v_{x}$ gradient, while the right, has negative and nearly constant gradient. Also in Fig. 2, we indicate that these two RNEMD regions correspond to regions of nearly constant vorticity, $\omega=\nabla \times \mathbf{v}$, although the directions of $\omega$ are in opposition in the two RNEMD regions. The vorticity on the left side of the simulation cell is a vector pointing along the positive $y$ axis, while on the right side, it points to the negative $y$ axis. This feature allows us to explore how enantiomers behave in regions of constant vorticity. 
In the lab-fixed frame, the opposing pitches of enantiomers in the same RNEMD region create opposite thrusts that are perpendicular to the $x z$-rotation plane, allowing separation and resolution of the enantiomers along the $y$-axis. ${ }^{23,28}$ As mentioned in above, this property is analogous to a screw with left-hand threads and its mirror image (right-hand threads) in which the medium is rotating around the screws. ${ }^{23,27-29}$

To measure the average separation of one enantiomer in the $y$-direction induced by shear vorticity, we computed a mean $y$ displacement,

$$
\langle\delta y(t)\rangle=\left\langle y_{i}(t+\tau)-y_{i}(\tau)\right\rangle_{i, \tau}
$$

for enantiomers $i$ in the left region (one of the colorless regions in Fig. 2), and another $\langle\delta y(t)\rangle$ for the same enantiomers in the right region. The correlations are averaged over initial times, $\tau$, and over the enantiomers that are present in the observation region. Note that molecules were included in these correlation functions as long as they stayed in a region of constant vorticity, i.e., in only one of the RNEMD regions. Observation was restarted for molecules which diffused to the other side of the box.

The results of individual simulations, one with $\Lambda-\left[\mathrm{Ru}(\mathrm{bpy})_{3}\right]^{2+}$ dissolved in liquid SPC/E water $^{37}$ solvent, and another simulation with $\Delta$-[Ru(bpy $\left.)_{3}\right]^{2+}$ dissolved in liquid SPC/E water ${ }^{37}$ solvent, show that these enantiomers migrate in opposite directions, according to their opposite pitches (see Fig. 3). The counterion $\mathrm{Cl}^{-}$is also present in the $\left[\mathrm{Ru}(\mathrm{bpy})_{3}\right]^{2+}$ simulations. Simulation parameters and details of the force fields and boxes employed are provided in the Supporting Information. 


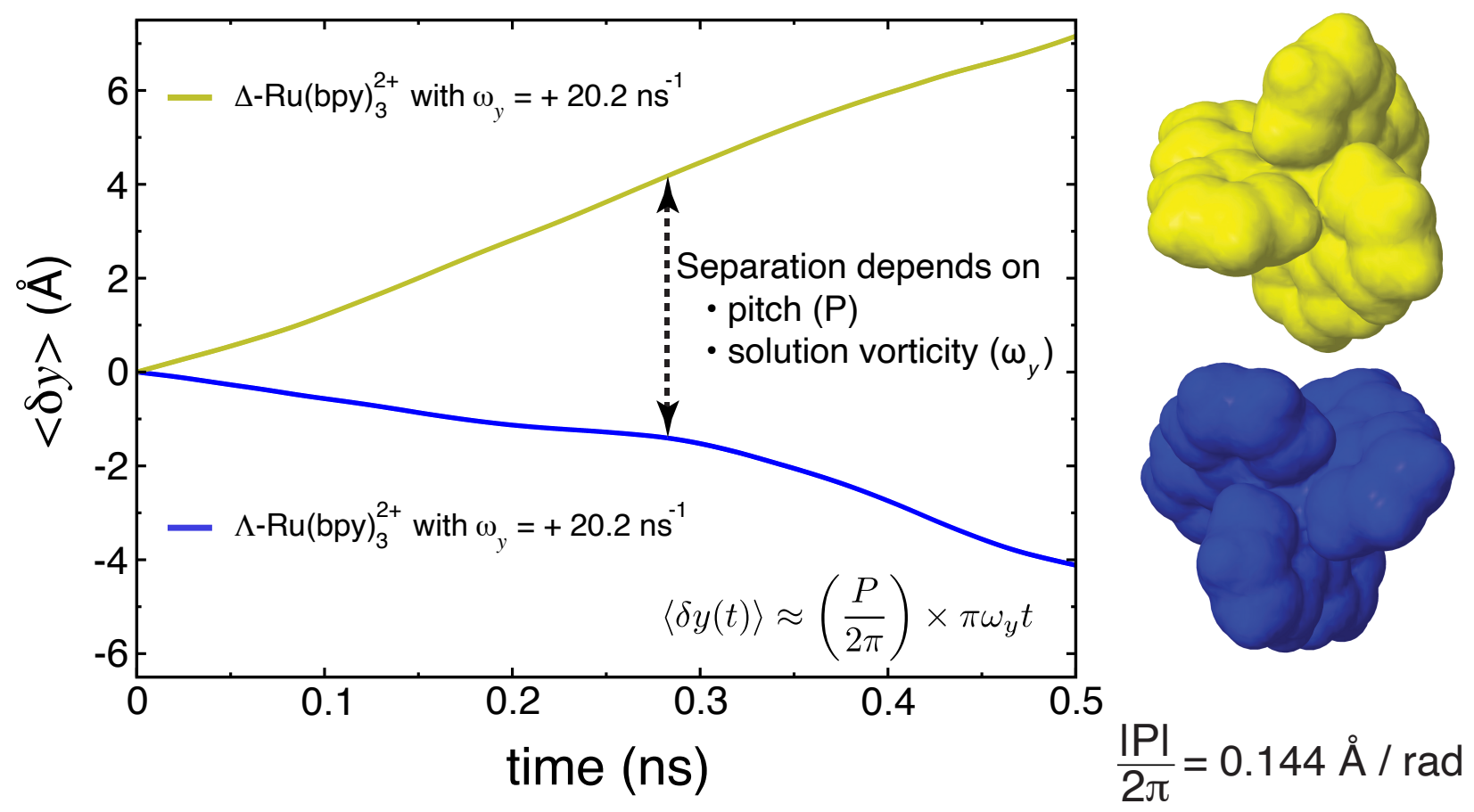

Figure 3: In regions of positive vorticity, isolated $\Lambda$ - $\left[\mathrm{Ru}(\mathrm{bpy})_{3}\right]^{2+}$ and $\Delta$-[Ru(bpy $\left.)_{3}\right]^{2+}$ enantiomers move in opposite directions from their initial positions. Molecules are included in the correlation function as long as they stay in a region of constant vorticity. Observation of that molecule restarts if it diffuses to the other side of the box. The approximate mean displacement can be estimated from solution vorticity and the definition of pitch in Eq. (3). 


\section{Pitch Values for Achiral Objects}

From the mirror image property of the translation-rotation coupling tensor $\left(\Xi^{\operatorname{tr}}\right)$, we have also discovered that achiral objects can also exhibit a non-zero molecular pitch. Indeed, any irregular, asymmetric body has non-vanishing translation-rotation coupling, but only some achiral objects can exhibit pitch as defined above. In the Supporting Information, we estimate the forward motion for a few achiral objects that exhibit pitch. Using the molecular pitch definition in Eq. (3), we predict very similar behavior to the experiments on ferromagnetic microparticle 'swimmers' that were recently propelled through a stationary fluid using magnetic rotation by Cheang et al. ${ }^{40}$

\section{Competition Between Enantiomers}

Consider a solution with right-handed screws $(R)$ and left-handed screws $(S)$ rotating in the $y$-axis under a constant revolution frequency. Since the screws are rigid bodies, their vorticity in the $\mathrm{y}$-axis $\left(\omega_{y}\right)$ is twice their revolution frequency. ${ }^{22,25,26}$ In this solution, there will be competition caused by perpendicular displacement of the screws: the $R$ screws will begin to move and pull their surroundings in the positive $y$-direction, while the $S$ screws will pull in the negative $y$-direction. A simple competition model uses the mole fractions of $R$ and $S$ to determine the resultant drift velocities of the screws and the surrounding solution. Using the definition of pitch, we can express the velocity of the solution,

$$
v_{\text {solution }}=\left(x_{R}-x_{S}\right)\left(\frac{|P|}{2 \pi}\right) \pi \omega_{y}
$$


and the velocity of the enantiomers,

$$
\begin{aligned}
& v_{R}=v_{\text {solution }}+x_{R}\left(\frac{|P|}{2 \pi}\right) \pi \omega_{y} \\
& v_{S}=v_{\text {solution }}-x_{S}\left(\frac{|P|}{2 \pi}\right) \pi \omega_{y}
\end{aligned}
$$

where $x_{R}=\frac{n_{R}}{n_{R}+n_{S}+n_{\text {solvent }}}$ is the mole fraction of the $R$ screws and $x_{S}$ is the mole fraction of the $S$ screws, and $n_{M}$ is the number of species $M$ present in the local volume.

Considering the relative velocities of $R$ and $S$, we can estimate the separation $(d)$ between the screws after a time interval $(t)$ :

$$
d=\left|\left\langle\delta y_{R}(t)\right\rangle-\left\langle\delta y_{S}(t)\right\rangle\right|=\frac{|P|}{2 \pi} \cdot \omega_{y} \pi t \cdot\left(x_{R}+x_{S}\right)
$$

We can also arrive at three limits, depending on whether the solvent interferes with the enantiomeric motion along $y$ (see Fig. 4):

1. Isolated enantiomers: when the solvent and the screws do not interfere with each other. In this case, $x_{R} \rightarrow 1$ in regions dominated by $R$ and $x_{S} \rightarrow 1$ in regions dominated by $S$.

2. Non-interfering solvent: when the motion of the enantiomers depends only on the concentrations of the two enantiomers, so $x_{R} \rightarrow\left(\frac{n_{R}}{n_{R}+n_{S}}\right)$ and $x_{S} \rightarrow\left(\frac{n_{S}}{n_{R}+n_{S}}\right)$.

3. Interfering solvent: when the solvent and the screws interfere with each other.

In Fig. 5, we apply the competition model developed here to the simulations of chiral molecules and solvents. We note that the chiral molecules and solvents simulated in this work appear to exist within the limits (1-3) of the competition model. 


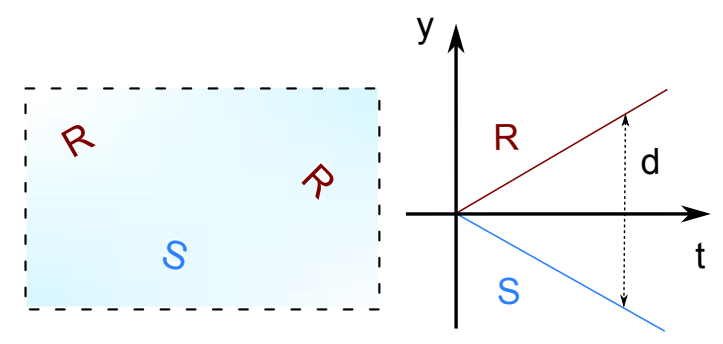

isolated enantiomers

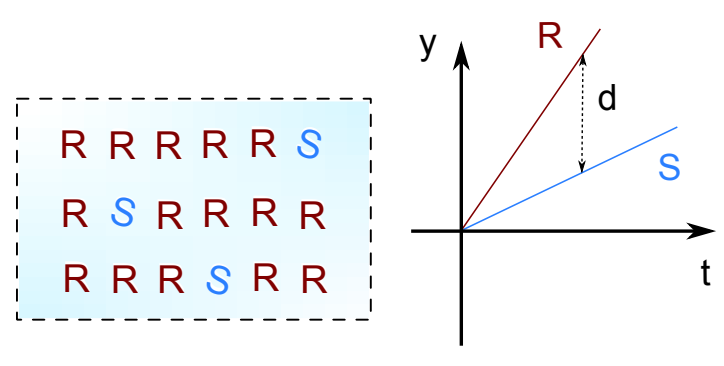

one enantiomer dominates

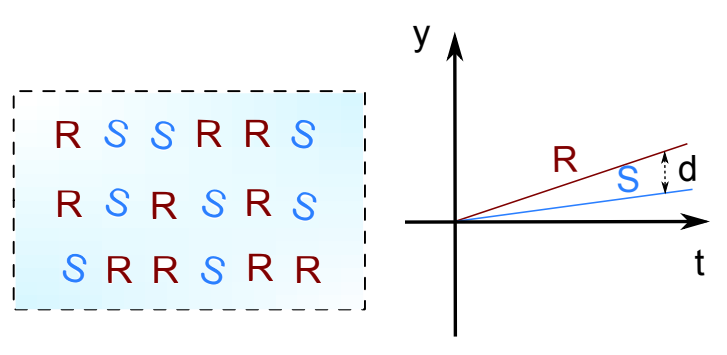

nearly racemic mixture

Figure 4: Competition between the local concentration of enantiomers determines both the background solvent velocity in the $y$-direction, as well as the separation $(d)$ of the two enantiomers. 


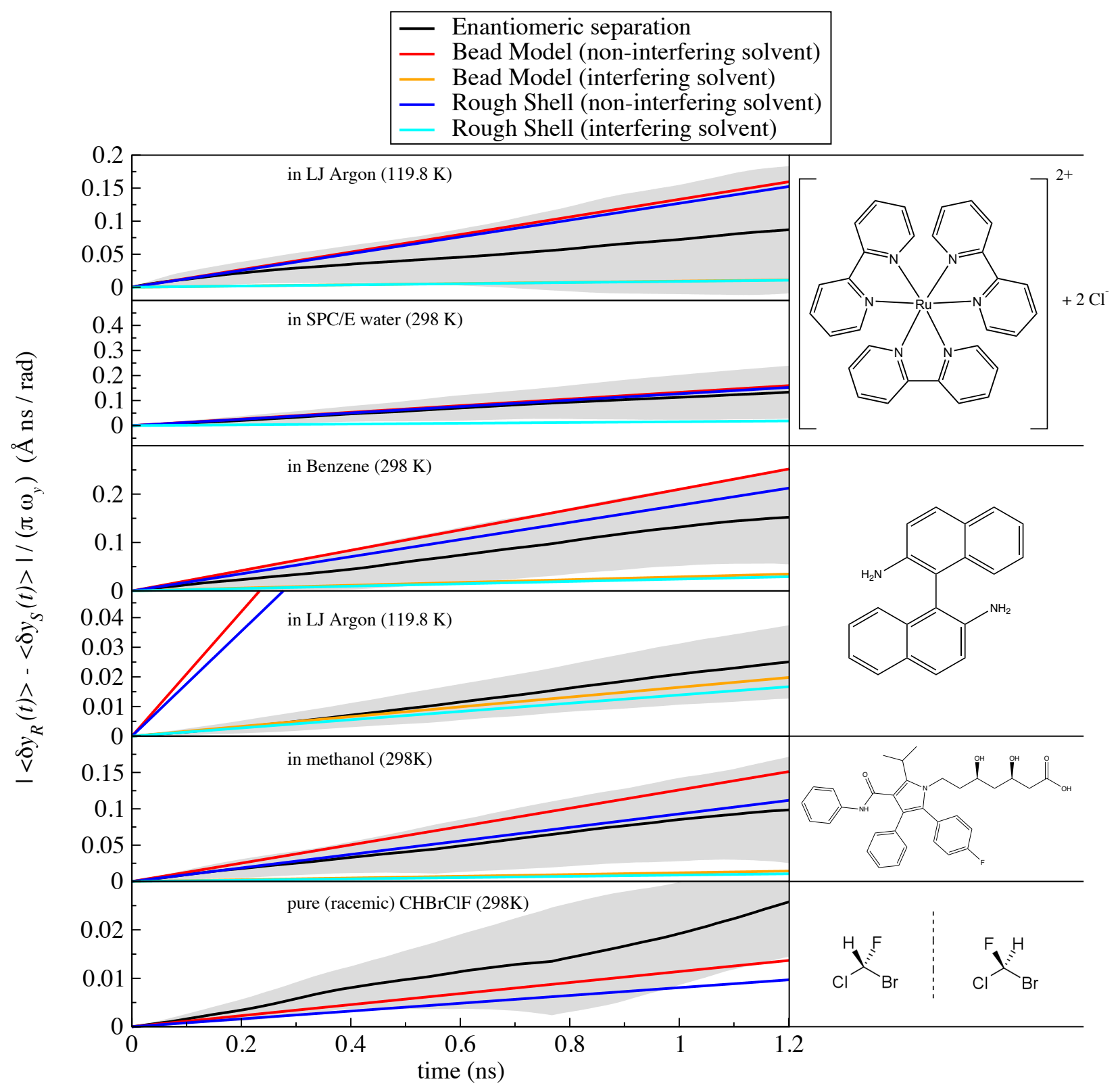

Figure 5: Mean enantiomeric separation (black lines) for a range of chiral molecules and solvents simulated under conditions of constant shear vorticity $\left(\omega_{y}\right)$. (Student's $t$-distribution) $95 \%$ confidence intervals ${ }^{26}$ around the separation data are shown in light grey. Predictions using Eq. (9) with molecular pitch values obtained from both bead and rough shell models are shown as straight colored lines. Note that there are no interfering solvent curves for the neat liquid $\mathrm{CHBrClF}$. 


\section{Coupled Drift-Diffusion Equations for Enantiomeric Mixtures}

We start with the one-dimensional drift-diffusion equation for a species $M,{ }^{41}$

$$
\frac{\partial c_{M}}{\partial t}=D_{\mathrm{tt}} \frac{\partial^{2} c_{M}}{\partial y^{2}}-v_{M} \frac{\partial c_{M}}{\partial y}
$$

where $c_{M}=c_{M}(y, t)$ is the concentration of $M$ as a function of the $y$-coordinate and the time $t$, $v_{M}$ is the constant $y$-velocity of $M$, and $D_{\mathrm{tt}}$ is the translational diffusion coefficient, which can be computed from the trace of its translational diffusion tensor. ${ }^{21,24,31}$ In the Supporting Information, we develop more fully the calculation of the molecular diffusion tensors.

For a nearly incompressible system, such as an aqueous solution of enantiomers $R$ and $S$, the volume $V_{o}$ and the number density, $\rho_{o}=\frac{n_{R}+n_{S}+n_{\text {solvent }}}{V_{o}}$ are nearly constant. With a constant vorticity, we can use Eq. (10) along with Eqs. (6)-(8) to combine our competition model with translational diffusion. This combination can be expressed in matrix form as coupled drift-diffusion equations for the $R$ and $S$ enantiomers:

$$
\frac{\partial}{\partial t}\left(\begin{array}{c}
c_{R} \\
c_{S}
\end{array}\right)=D_{\mathrm{tt}} \frac{\partial^{2}}{\partial y^{2}}\left(\begin{array}{c}
c_{R} \\
c_{S}
\end{array}\right)-\frac{|P| \omega_{y}}{2 \rho_{o}}\left(\begin{array}{cc}
2 & -1 \\
1 & -2
\end{array}\right)\left(\begin{array}{c}
c_{R} \\
c_{S}
\end{array}\right) \frac{\partial}{\partial y}\left(\begin{array}{c}
c_{R} \\
c_{S}
\end{array}\right)
$$

Note that the other limits of the competition model, isolated enantiomers and non-interfering solvents, can be obtained easily by rewriting $\rho_{o}$ in terms of the density of the enantiomers instead of the density of the solution, $\rho_{o}=\frac{n_{R}+n_{S}}{V_{o}}$.

Translational diffusion has the effect of mixing the enantiomers, opposing the shear-driven separation. With Eq. (11) and with initial concentrations for the enantiomer $R$ and $S$, we can estimate the feasibility of the separation through the following variables: molecular pitch $(P)$, a property of the enantiomer itself; vorticity $(\omega)$, an experimental condition; density $\left(\rho_{o}\right)$, a solution property; and the translational diffusion coefficient $\left(D_{\mathrm{tt}}\right)$, a molecular property that varies with temperature and solution viscosity.

In Fig. 6, we show the concentration profiles after injection of a racemic mixture into the center 
of a region of constant vorticity. On a timescale of hours, the vortex (shear) flow is predicted to separate the two enantiomers on a centimeter length scale. In this figure, we have used parameters that mimic drug-like enantiomeric molecules in a dense fluid.

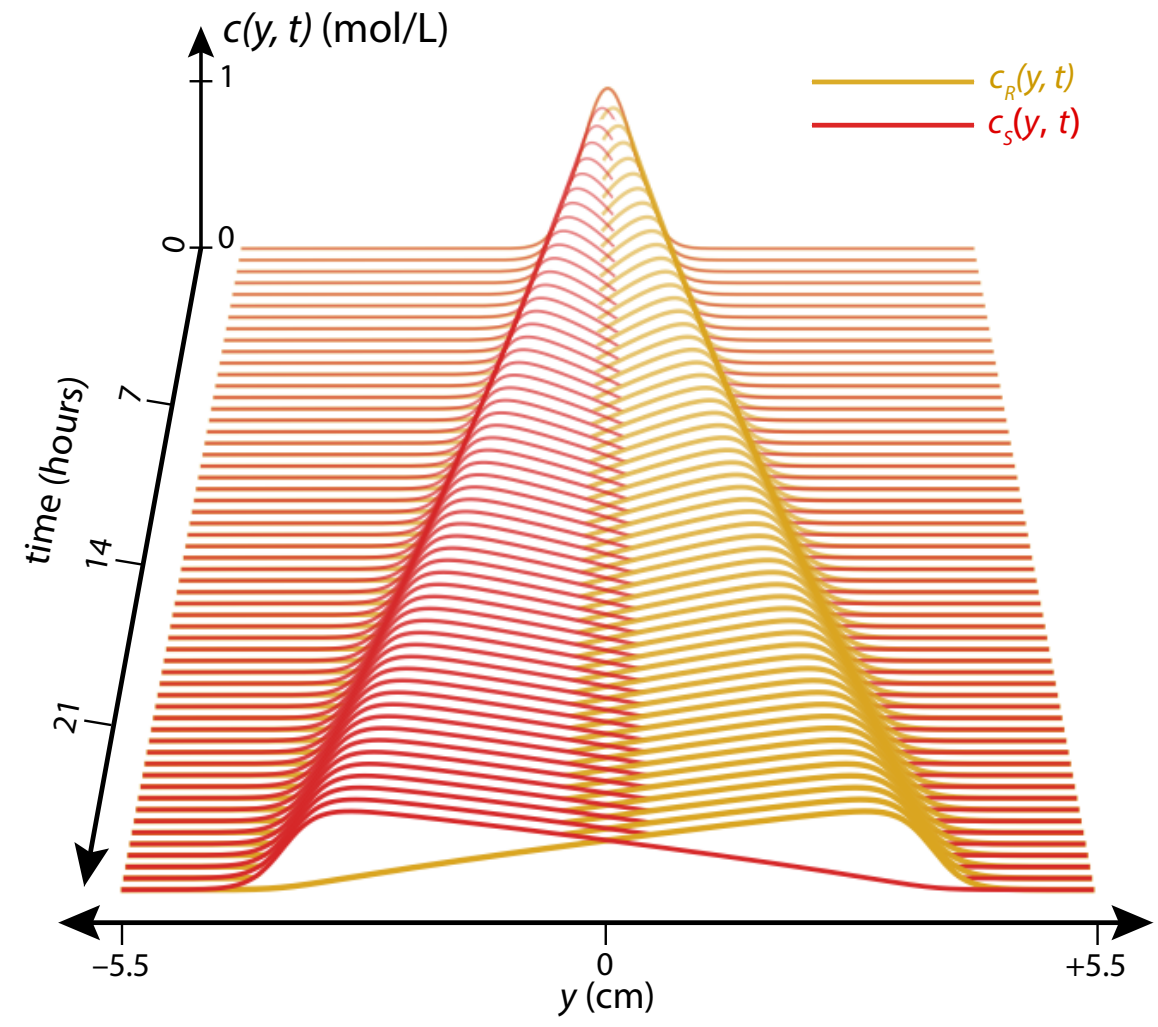

Figure 6: Enantiomeric separation of a racemic mixture using vortex flow. A racemic mixture with a Gaussian concentration profile is injected at $y=0$ and $t=0$. Under a vorticity, $\omega=1.6 \times 10^{6} \mathrm{~s}^{-1}$, and a translational diffusion constant, $D_{\mathrm{tt}}=5 \times 10^{-6} \mathrm{~cm}^{2} \mathrm{~s}^{-1}$, the two enantiomers separate on a centimeter length scale in a few hours. Each successive curve is 30 minutes later than the curve above. In this figure, the enantiomers have a molecular pitch, $|P| / 2 \pi=0.1 \AA \mathrm{rad}^{-1}$, and a solution density, $\rho_{o}=100 \mathrm{~mol} / \mathrm{L}$.

In the Supporting Information, we show results for a wide range of translational diffusion constants. For small $D_{\mathrm{tt}}$, separation appears to be possible with sufficient solution vorticity. The tradeoff between the solution and molecular properties on the right-hand side of Eq. (11) can be expressed in a simple ratio,

$$
\frac{|P| \omega_{y}}{2 \rho_{o} D_{\mathrm{tt}}} \gtrsim 1 .
$$

Our drift-diffusion simulations indicate that when this ratio exceeds $1 \mathrm{~L} \mathrm{~mol}^{-1} \mathrm{~cm}^{-1}$, racemic mixtures do eventually separate on a centimeter length scale. Further details on the drift-diffusion 
simulations are provided in the Supporting Information.

\section{Discussion and Conclusions}

Given the theory and numerical simulations described here, enantiomeric separation should be possible when the ratio in Eq. (12) exceeds $1 \mathrm{~L} \mathrm{~mol}^{-1} \mathrm{~cm}^{-1}$. The majority of the drug-like enantiomeric molecules studied here have $|P| / 2 \pi$ on the order of $0.1 \AA / \mathrm{rad}$. In table 2 , taking $\rho_{o} \approx 100$ $\mathrm{mol} / \mathrm{L}$ for a high density solution, we have estimated both the ratio in Eq. (12) and the required separation time for molecules with a wide range of translational diffusion constants.

Table 2: Practical limits for vorticity-induced enantiomeric separation, using a number density, $\rho_{o}=100 \mathrm{~mol} \mathrm{~L}^{-1}$, and a molecular pitch, $|P| /(2 \pi)=0.1 \AA / \mathrm{rad}$. Translational diffusion can make it impossible to separate enantiomers unless the vorticity is large enough to overcome diffusive mixing. Note that large molecules in water $\left(\rho_{o} \approx 55.5 \mathrm{~mol} \mathrm{~L}^{-1}\right)$ typically have diffusion constants in the range: ${ }^{42} 10^{-5}-10^{-6} \mathrm{~cm}^{2} \mathrm{~s}^{-1}$.

\begin{tabular}{c|c|c|c}
\hline$D_{\mathrm{tt}}\left(\mathrm{cm}^{2} \mathrm{~s}^{-1}\right)$ & $\omega_{y}\left(s^{-1}\right)$ & $\frac{|P| \omega_{y}}{2 \rho_{o} D_{\mathrm{tt}}}\left(\mathrm{L} \mathrm{mol}^{-1} \mathrm{~cm}^{-1}\right)$ & Time required for separation $(\mathrm{s})$ \\
\hline \multirow{5}{*}{$5 \times 10^{-4}$} & $1.6 \times 10^{4}$ & $10^{-3}$ & No separation \\
& $1.6 \times 10^{5}$ & $10^{-2}$ & No separation \\
& $1.6 \times 10^{6}$ & $10^{-1}$ & No separation \\
& $1.6 \times 10^{7}$ & 1 & $10^{3}-10^{4}$ \\
& $1.6 \times 10^{8}$ & $10^{1}$ & $10^{2}-10^{3}$ \\
\hline \multirow{5}{*}{$5 \times 10^{-5}$} & $1.6 \times 10^{4}$ & $10^{-2}$ & No separation \\
& $1.6 \times 10^{5}$ & $10^{-1}$ & No separation \\
& $1.6 \times 10^{6}$ & 1 & $10^{4}-10^{5}$ \\
& $1.6 \times 10^{7}$ & $10^{1}$ & $10^{3}-10^{4}$ \\
& $1.6 \times 10^{8}$ & $10^{2}$ & $10^{2}-10^{3}$ \\
\hline \multirow{3}{*}{$5 \times 10^{-6}$} & $1.6 \times 10^{4}$ & $10^{-1}$ & No separation \\
& $1.6 \times 10^{5}$ & 1 & $10^{5}-10^{6}$ \\
& $1.6 \times 10^{6}$ & $10^{1}$ & $10^{4}-10^{5}$ \\
& $1.6 \times 10^{7}$ & $10^{2}$ & $10^{3}-10^{4}$ \\
& $1.6 \times 10^{8}$ & $10^{3}$ & $10^{2}-10^{3}$ \\
\hline \hline
\end{tabular}

For any relatively rigid enantiomeric molecule, the formalism presented here allows computation of the molecular pitch. Once a solvent is selected, both the number density and translational 
diffusion constant can be estimated. All that remains, then, is to find a vorticity that would make separation possible on the centimeter scale.

Because most molecules in water $\left(\rho_{o} \approx 55.5 \mathrm{~mol} \mathrm{~L}^{-1}\right)$ typically have diffusion constants in the range: $10^{-5}-10^{-6} \mathrm{~cm}^{2} \mathrm{~s}^{-1},{ }^{42}$ it appears that vortex separation of enantiomers on a centimeter scale should be experimentally achievable, even under conditions where the enantiomers are in competition in a racemic mixture.

We note that although we have used shear flow as the source to rotate the chiral molecules and achieve separation, it may be possible to use external forces to rotate the enantiomers. One example would be to use a rotating field which couples to the electric dipole moment of the molecule. ${ }^{34,43,44}$ The separation mechanism in a fluid should follow the same theory developed here.

There are two aspects of this work which are ripe for future exploration. One involves the role of molecular flexibility in vortex separation. Molecular flexibility makes pitch a dynamic property of the molecule, and could yield nonlinear responses to constant-vortex flow. Predicting separation of flexible molecules may be a simple matter of combining multiple pitch values from multiple conformations, but we do not yet have the data to predict whether a dynamic pitch will enhance or reduce vortex separation of enantiomers. A second aspect that merits further exploration is the role of dynamic friction. Solvents which respond slowly to perturbations from the embedded enantiomers may require the use of the generalized Langevin equation (GLE), which utilizes timedependent friction kernels that retain memory of previous perturbations in the fluid. This would certainly complicate the picture of resistance tensors and molecular pitch. Further investigation of how this would alter both the screw model and the separation dynamics are warranted.

\section{Supporting Information Available}

The supporting documentation contains hydrodynamic and Green-Kubo derivations of the resistance and diffusion tensors, theoretical development of the overlapping bead and rough shell models for the resistance tensors, a version of the molecular pitch using diffusion tensors, screw model 
pitch predictions for 85 drug-like molecules, details and simulation parameters for the RNEMD and coupled drift-diffusion simulations, and an examination of pitch values for certain achiral molecules that naturally emerge from mirror image properties of the hydrodynamic tensors. A accompanying set of text files provide the rigid body molecular geometries and force field parameters for all molecules (and solvents).

\section{Acknowledgement}

Support for this project was provided by the National Science Foundation under grants CHE1663773 and CHE-1954648. Computational time was provided by the Center for Research Computing (CRC) at the University of Notre Dame.

\section{References}

(1) McNaught, A. D., Wilkinson, A., Eds. IUPAC. Compendium of Chemical Terminology, (the "Gold Book”), 2nd ed.; Blackwell Scientific Publications: Oxford, 1997; Online version (2019-) created by S. J. Chalk.

(2) Jacques, J.; Collet, A.; Wilen, S. H. Enantiomers, Racemates, and Resolutions, 1st ed.; John Wiley \& Sons, Inc.: New York, 1981.

(3) Todd, M. Introduction: A Survey of How and Why to Separate Enantiomers. In Separation of Enantiomers: Synthetic Methods; Todd, M., Ed.; John Wiley \& Sons, Ltd, 2014; pp 1-12.

(4) Nguyen, L. A.; He, H.; Pham-Huy, C. Chiral Drugs: An Overview. Int. J. Biomed. Sci. 2006, 2, 85-100.

(5) Pavia, D. L.; Lampman, G. M.; Kriz, G. S.; Engel, R. G. A Small Scale Approach to Organic Laboratory Techniques, 3rd ed.; Brooks/Cole, Cengage Learning: Belmont, CA, 2011. 
(6) Carey, J. S.; Laffan, D.; Thomson, C.; Williams, M. T. Analysis of the Reactions Used for the Preparation of Drug Candidate Molecules. Org. Biomol. Chem. 2006, 4, 2337-2347.

(7) Görög, S.; Gazdag, M. Enantiomeric Derivatization for Biomedical Chromatography. J. Chromatogr. B Biomed. Sci. Appl. 1994, 659, 51-84.

(8) Holton, J. R. In An Introduction to Dynamic Meteorology, 4th ed.; Dmowska, R., Holton, J. R., Thomasrossby, H., Eds.; International Geophysics Series; Elsevier Academic Press: Burlington, MA, 2004; Vol. 88.

(9) Lackmann, G. Midlatitude Synoptic Meteorology: Dynamics, Analysis, and Forecasting; American Meteorological Society: Boston, MA, 2011.

(10) Howard, D. W.; Lightfoot, E. N.; Hirschfelder, J. O. The Hydrodynamic Resolution of Optical Isomers. AIChE J. 1976, 22, 794-798.

(11) Hirschfelder, J. O.; Lightfoot, E. N.; Howard, D. W. Hydrodynamic Method for Separation of Solid Bodies or Crystals. U.S. Patent 4,010,095, issued March 1, 1977.

(12) Makino, M.; Arai, L.; Doi, M. Shear Migration of Chiral Particle in Parallel-Disk. J. Phys. Soc. Jpn. 2008, 77, 064404.

(13) Hermans, T. M.; Bishop, K. J. M.; Stewart, P. S.; Davis, S. H.; Grzybowski, B. A. Vortex Flows Impart Chirality-Specific Lift Forces. Nat. Commun. 2015, 6, 5640.

(14) Marcos,; Fu, H. C.; Powers, T. R.; Stocker, R. Separation of Microscale Chiral Objects by Shear Flow. Phys. Rev. Lett. 2009, 102, 158103.

(15) Aristov, M.; Eichhorn, R.; Bechinger, C. Separation of Chiral Colloidal Particles in a Helical Flow Field. Soft Matter 2013, 9, 2525-2530.

(16) Ro, S.; Yi, J.; Kim, Y. W. Chiral Separation by Flows: The Role of Flow Symmetry and Dimensionality. Sci. Rep. 2016, 6, 35144. 
(17) Schamel, D.; Pfeifer, M.; Gibbs, J. G.; Miksch, B.; Mark, A. G.; Fischer, P. Chiral Colloidal Molecules And Observation of The Propeller Effect. J. Am. Chem. Soc. 2013, 135, 1235312359.

(18) Alcanzare, M. M. T.; Thakore, V.; Ollila, S. T. T.; Karttunen, M.; Ala-Nissila, T. Controlled Propulsion and Separation of Helical Particles at the Nanoscale. Soft Matter 2017, 13, 21482154.

(19) Tencer, M.; Bielski, R. Mechanical Resolution of Chiral Objects in Achiral Media: Where is the Size Limit? Chirality 2011, 23, 144-147.

(20) Marichez, V.; Tassoni, A.; Cameron, R. P.; Barnett, S. M.; Eichhorn, R.; Genet, C.; Hermans, T. M. Mechanical Chiral Resolution. Soft Matter 2019, 15, 4593-4608.

(21) Landau, L. D.; Lifshitz, E. M. Fluid Mechanics, 2nd ed.; Course of Theoretical Physics; Pergamon Press: Oxford, England, 1987; Vol. 6; translated from the Russian by J. B. Sykes and W. H. Reid.

(22) Tritton, D. J. Physical Fluid Dynamics, 2nd ed.; Clarendon Press; Oxford University Press: Oxford, England, UK, 1988.

(23) Brenner, H. The Stokes Resistance of an Arbitrary Particle-II: An Extension. Chem. Eng. Sci. 1964, 19, 599-629.

(24) Harvey, S.; Garcia de la Torre, J. Coordinate Systems for Modeling the Hydrodynamic Resistance and Diffusion Coefficients of Irregularly Shaped Rigid Macromolecules. Macromolecules 1980, 13, 960-964.

(25) Khan, M. K. Fluid Mechanics and Machinery; Oxford University Press: Oxford, England, UK, 2015.

(26) Riley, K. F.; Hobson, M. P.; Bence, S. J. Mathematical Methods for Physics and Engineering, 
3rd ed.; Cambridge University Press: Cambridge, UK, 2006; pp 241-315, 334-376, 12211304.

(27) Ball, R. S. The Theory of Screws: A Study in the Dynamics of a Rigid Body; Hodges, Foster and Co.: Dublin, 1876.

(28) Baranova, N. B.; Zel'dovich, B. Y. Separation of Mirror Isomeric Molecules by RadioFrequency Electric Field of Rotating Polarization. Chem. Phys. Lett. 1978, 57, 435-437.

(29) Bhandari, V. B. Design of Machine Elements, 3rd ed.; Tata McGraw-Hill Education Private Ltd.: New Delhi, 2010; pp 184-271.

(30) García de la Torre, J.; Bloomfield, V. A. Hydrodynamic Properties of Macromolecular Complexes. I. Translation. Biopolymers 1977, 16, 1747-1763.

(31) Carrasco, B.; García de la Torre, J. Hydrodynamic Properties of Rigid Particles: Comparison of Different Modeling and Computational Procedures. Biophys. J. 1999, 76, 3044-3057.

(32) Sun, X.; Lin, T.; Gezelter, J. D. Langevin Dynamics for Rigid Bodies of Arbitrary Shape. J. Chem. Phys. 2008, 128, 234107.

(33) Zuk, P. J.; Wajnryb, E.; Mizerski, K. A.; Szymczak, P. Rotne-Prager-Yamakawa Approximation for Different-Sized Particles in Application to Macromolecular Bead Models. J. Fluid Mech. 2014, 741, R5.

(34) Kibar, O.; Chachisvilis, M.; Tu, E.; Marsilje, T. H. Separation and Manipulation of a Chiral Object. U.S. Patent 8,698,031 B2, issued April 15, 2014.

(35) Josefsson, I.; Eriksson, S. K.; Rensmo, H.; Odelius, M. Solvation Structure around Ruthenium(II) Tris(bipyridine) in Lithium Halide Solutions. Struct. Dyn. 2016, 3, 023607.

(36) Zelewsky, A. v. Stereochemistry of Coordination Compounds; Inorganic Chemistry: A Textbook Series; John Wiley \& Sons: Chichester, England, 1996. 
(37) Berendsen, H. J. C.; Grigera, J. R.; Straatsma, T. P. The Missing Term in Effective Pair Potentials. J. Phys. Chem. 1987, 91, 6269-6271.

(38) Kuang, S.; Gezelter, J. D. Velocity Shearing and Scaling RNEMD: a Minimally Perturbing Method for Simulating Temperature and Momentum Gradients. Mol. Phys. 2012, 110, 691701.

(39) Kothandaraman, C. P.; Rudramoorthy, R. Fluid Mechanics and Machinery, 3rd ed.; New Academic Science Ltd.: London, England, UK, 2013.

(40) Cheang, U. K.; Meshkati, F.; Kim, D.; Kim, M. J.; Fu, H. C. Minimal Geometric Requirements for Micropropulsion via Magnetic Rotation. Phys. Rev. E 2014, 90, 033007.

(41) McQuarrie, D. A. Statistical Mechanics; Harper's Chemistry Series; Harper \& Row Publishers: New York, 1976.

(42) Rumble, J. R., Ed. Diffusion Coefficients in Liquids at Infinite Dilution. In CRC Handbook of Chemistry and Physics, 101st ed.; CRC Press/Taylor \& Francis: Boca Raton, FL, 2020; Online version 2020.

(43) Clemens, J. B.; Kibar, O.; Chachisvilis, M. A Molecular Propeller Effect for Chiral Separation and Analysis. Nat. Commun. 2015, 6, 7868.

(44) Chachisvilis, M. Chiral Analysis and Separation Using Molecular Rotation. In Chiral Analysis: Advances in Spectroscopy, Chromatography and Emerging Methods, 2nd ed.; Polavarapu, P. L., Ed.; Elsevier: Cambridge, MA, 2018; pp 753-778. 


\section{TOC Graphic}

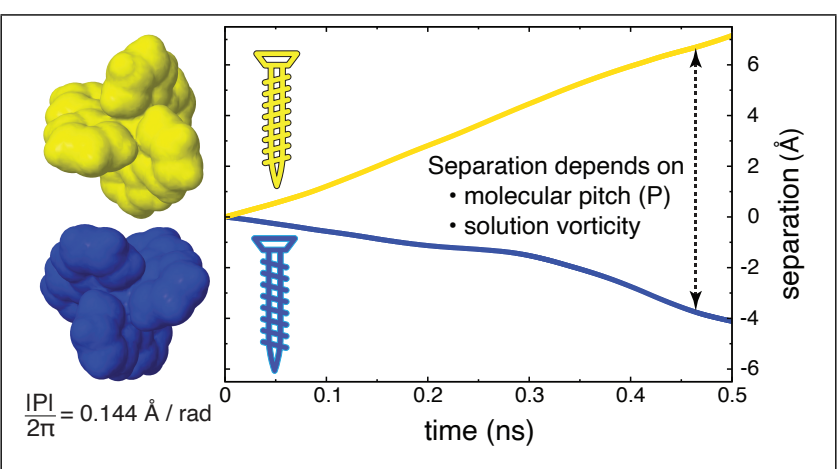

